\title{
Successful Handling of Covid-19 in a Young Patient with SCID due to CARMIL2 (RLTPR) Deficiency: Case Report and Review of Literature
}

\author{
Khaled Mohammed Al-Qahtani ${ }^{1}$, Ali Saleh Alhamdan ${ }^{2}$ Wael F \\ Alblowi $^{3 *}$, Mohammed Manei Al-Amri ${ }^{4}$, Mohammed Hasan Almalki ${ }^{5}$ \\ ${ }^{1,2}$ Department of Internal Medicine, Resident of internal Medicine at King Saud \\ Medical City. \\ ${ }^{3}$ Department of Internal Medicine, Medical Consultant, Allergist and Clinical \\ Immunologist at King Saud Medical City. \\ ${ }^{4}$ Department of Internal Medicine, Consultant of Internal Medicine and at King \\ Saud Medical City. \\ ${ }^{5}$ Department of Internal Medicine, Medical Consultant and toxicologist at King \\ Saud Medical City.
}

*Corresponding Author: Wael F Alblowi, Department of Internal Medicine, Medical

Consultant, Allergist and Clinical Immunologist at King Saud Medical City.

\begin{abstract}
The authors herein report a case of severe combined immunodeficiency (SCID) secondary to a rare genetic mutation of CARMIL2. Presented initially as a case of celiac disease then further investigated to reach the diagnosis of SCID secondary to CARMIL2. Furthermore, the patient has got infected by the recent pandemic COVID-19 and surprisingly passed through the infection asymptomatically. Consequently, discussion regarding of the association and pathophysiology of SCID and COVID-19 and how the underlying disease affected the results of the course of the disease. Interestingly, in our case the rationale of asymptomatic presentation of the disease is not clearly explained in the current literature whether its due to escape of the cytokine storm that the infection causes. Our hypothesis is that due to the severe immunodeficiency in our patient, it evidently helped her to avoid dysregulated immune responses to the virus and escape possible immunopathology status.
\end{abstract}

Keywords: Primary Immunodeficiency (PID); CARMIL2; COVID-19

\section{Introduction}

Primary immunodeficiency (PID) is a heterogenous group of disorders manifests in decrease or absence of parts of the immune system, classified into different categories depending on which part it manifests. T-cell immunodeficiency, B-cell immunodeficiency, combined immunodeficiency (CID) and disorders of innate immunity are the general categorization of PID [7].

Combined immunodeficiency disorder (CID) on its own words is defined as deficiency in both $\mathrm{T}$ and $\mathrm{B}$ lymphocyte where most
Received: August 15, 2020

Published: August 31, 2020

(C) All rights are reserved by Wael F Alblowi., et al. 
of this disorder is a primary autosomal recessive human immunodeficiency in CARMIL2 which is responsible for CD28 co-signaling and cytoskeletal dynamics necessary for $\mathrm{T}$ development, activation, proliferation, differentiation and function [2].

Most of the patients (89\%) with this disorder initially presents in the first 6 months of age manifesting as recurrent pneumonia (66\%), failure to thrive (60\%) and chronic diarrhea (35\%). In addition, consanguineous relationships tend to have higher incidence of autosomal recessive SCID.

Knowing the nature of this disorder, treatment modality of those patients is bone marrow transplant (BMT) and/or transfusing intravenous immunoglobulins to keep them circulating in their system while giving proper prophylactic antibiotics and antivirals to defend against recurrent and opportunistic infections [1]. SCID has the poorest prognosis among combined immunodeficiencies.

COVID-19 as of its predecessors is a zoonic viral infection such as a MERS-COV and SARS. It is currently an unprecedent pandemic that has rose up to us due to its contagious nature and lack of potent and efficient management and prevention plan [5].

The nature of this zoonic viral disease similar to its predecessors that it mainly manifests as a viral pneumonia causing damage to the alveoli of the lungs with its common complication of superinfection of bacterial pneumonia, also cytokine storm. In light of this information, manifestations of COVID-19 are usually pneumonia like symptoms such as dyspnea, cough, and fever. The severity of the disease is quite varied from asymptomatic to ventilator dependent patients. Unfortunately, with deficient information of the disease, we don't have a clear notion of how the virus manifests and depicts its symptoms [6]. Knowingly, studies of COVID-19 with its association with other diseases and/or different immunological status of patients should be investigated.

In summary, researches around the world should be encouraged regarding COVID-19 with other associated diseases.

\section{Case Presentation}

The authors are reporting a 14 years old Syrian girl known case of celiac disease, with recurrent infections since childhood. Presented to the outpatient clinic complaining of abdominal pain, dysphagia and failure to thrive. In this instance, investigations have been initiated to find out the cause.
CT abdomen and endoscopy were done showing bilateral adrenal mass and duodenal ulcer positive for CMV virus, H. Pylori and candida infections. While there is possibility for EBV to be the cause of this adrenal mass. Patient underwent surgery, laparoscopic Rt adrenalectomy and a punch biopsy of the left adrenal, both results revealed bilateral adrenal EBV associated smooth muscle tumor.

In the light of information and whole presentation of the case in regards of her past medical history, initial presentation and investigations aroused the suspicion of immunodeficiency disease. Genetic testing was done, and result concluded with a diagnosis of autosomal recessive CARMIL 2 syndrome related to combined immunodeficiency. Thus, patient started on monthly IVIG, and prophylaxis dose of Co-Trimoxazole and Valganciclovir, with a good improvement regarding recurrent infections, diet and growth.

During the last month patient was positive for COVID 19, she got the infection from her family and her father was severely ill and admitted to ICU, her mother and 2 siblings were also infected but their symptoms were mild and treated symptomatically with home isolation.

The patient was asymptomatic, and she was in tele contact with her primary immunological Consultant. She is a known case of SCID, although, interestingly she survived through this without any symptoms unlike her family, she was re-swabbed after 17 days from the first swab and it turned out negative.

\section{Discussion}

The authors report a case of a patient with severe combined immunodeficiency accompanied with its multiple coinfections and complications while taking all precautions and medications of intravenous immunoglobulins and prophylactic antibiotics. In our case, the patient has a rare genetic mutation for her SCID which is CARMIL2 deficiency. In one study they found the most common clinical finding in CARMIL2-deficient patients was EBV+ SMT which is relevant to our case. EBV+ SMT is a rare tumor commonly associated with secondary immunodeficiency disorder, for example, HIV-infection or immunosuppression patient in organ transplant patients [8].

In the current crisis going on, our patient got infected by recent surfaced pandemic COVID-19 due to contact with an infected relative. During the disease, our patient was completely asymptomatic unlike her relative who was admitted in the ICU which bears the 
question if the immune status of our patient helped during this infection.

A study has found that there is high 1.55 -fold increased risk of severe COVID-19 disease associated with immunodeficient patients ( 5 studies, 3,231 patients; OR $=1.55$, 95\%CI: 0.70 to 3.45 , $\mathrm{P}=0.285 ; \mathrm{I}^{2}=0.0 \%$ ). Although, there is no significant statistical difference3. In addition, another study reported in 1,590 patients with immunodeficiency associated with COVID-19 between dead and surviving revealed that there was no correlation in the risk of death in patients with both immunodeficiency and the disease $(\mathrm{P}=$ 1.000) [3]. Thus, the presentation in our case being asymptomatic concludes that presumably the risk of severity is not entirely correlated with the immunological status.

On the other hand, there is accumulating evidence suggests that groups who are infected with severe COVID-19 develop into having a cytokine storm syndrome, where the immune system goes into hyperinflammation [10]. To dig deeper into what we know about the immune status of COVID-19, the infection engages initially on pattern-recognition receptor (PRRs) pathway via RIG-I like receptors (RLRs) and endosomal Toll-like receptors (TLRs) which cascades into the secretion of cytokines among them the type I/ III interferons (IFNs) are considered most important for antiviral defense 9 and it can effectively limit the virus infection. On the other hand, to add upon this information, research suggests that the virus developed ways to escape the immunity by dysregulating the IFNs pathway in response to its pathogenicity 10 which correlates with the severity of the disease. In addition, dysregulated immune responses of the infection would in evidently cause an immunopathology [11].

Interestingly, in our case the rationale of asymptomatic presentation of the disease is not clearly explained in the current literature whether its due to escape of the cytokine storm that the infection causes. Our hypothesis is that due to the severe immunodeficiency in our patient, it evidently helped her to avoid dysregulated immune responses to the virus and escape possible immunopathology status.

Within this pandemic there is lack of information regarding the immune response to COVID-19 and its pathophysiological features, debating whether to continue with immunoglobulin substitutions for immunological deficient patients, in a study it has been recommended to continue with the immunoglobulin substitutions and the possible use of convalescent plasma in these patients [4]. It has been shown that cytotoxic lymphocytes which essential in control of the virus mechanism is markedly decreased in numbers both NK and $\mathrm{CD}^{+} \mathrm{T}$ cell function.

\section{Conclusion}

Patients with immunological deficiencies who are at risk of COVID-19 or infected by it, should continue their treatment regimen and follow-up with their attending physician via telemedicine, that is the current recommendation [4]. Furthermore, there is lack of information regarding the immunological mechanisms with $\mathrm{CO}$ VID-19 which is a good area of research especially there are a lot of different immunological status for patients at risk.

\section{Bibliography}

1. Justiz Vaillant AA and Mohseni M. "Severe Combined Immunodeficiency”. In: StatPearls. Treasure Island (FL): StatPearls Publishing (2020).

2. T Schober., et al. "A human immunodeficiency syndrome caused by mutations in CARMIL2". Nature Communication 8 (2017).

3. Gao Y., et al. "Impacts of immunosuppression and immunodeficiency on COVID-19: A systematic review and meta-analysis". Journal of Infection 81.2 (2020): e93-e95.

4. Brough HA., et al. "Managing childhood allergies and immunodeficiencies during respiratory virus epidemics - The 2020 COVID-19 pandemic: A statement from the EAACI section on pediatrics". Pediatric Allergy and Immunology (2020).

5. Liu J., et al. "Overlapping and discrete aspects of the pathology and pathogenesis of the emerging human pathogenic coronaviruses SARS-CoV, MERS-CoV, and 2019-nCoV". Journal of Medical Virology 92 (2020): 491-494.

6. Pascarella G., et al. "COVID-19 diagnosis and management: a comprehensive review". Journal of Internal Medicine 288.2 (2020): 192-206.

7. Geha RS., et al. "International Union of Immunological Societies Primary Immunodeficiency Diseases Classification Committee". Journal of Asthma and Clinical Immunology 120.4 (2007): 776-794.

8. T Schober., et al. "A human immunodeficiency syndrome caused by mutations in CARMIL2". Nature Communication (2017). 
9. Mehta P., et al. “COVID-19: consider cytokine storm syndromes and immunosuppression”. Lancet 395.10229 (2020): 10331034.

10. Channappanavar R., et al. "Dysregulated Type I Interferon and Inflammatory Monocyte-Macrophage Responses Cause Lethal Pneumonia in SARS-CoV-Infected Mice". Cell Host and Microbes 19 (2016): 181-193.

11. McCusker $\mathrm{C}$ and Warrington R. "Primary immunodeficiency". Allergy, Asthma and Clinical Immunology 1 (2011): S11.

\section{Assets from publication with us}

- Prompt Acknowledgement after receiving the article

- Thorough Double blinded peer review

- Rapid Publication

- Issue of Publication Certificate

- High visibility of your Published work

Website: $\underline{w w w}$.actascientific.com/

Submit Article: www.actascientific.com/submission.php

Email us: editor@actascientific.com

Contact us: +919182824667 